\title{
Comparison of advanced adenocarcinomas of esophagogastric junction and distal stomach in Japanese patients
}

\author{
Akiko Kawano · Takako Eguchi Nakajima - Ichiro Oda • Nobukazu Hokamura • \\ Satoru Iwasa - Ken Kato · Tetsuya Hamaguchi • Yasuhide Yamada • \\ Hirofumi Fujii · Yasuhiro Shimada
}

Received: 30 January 2012/Accepted: 3 February 2013/Published online: 24 February 2013

(c) The International Gastric Cancer Association and The Japanese Gastric Cancer Association 2013

\begin{abstract}
Background There have been no reports on the incidence, characteristics, treatment outcomes, and prognosis of inoperably advanced or recurrent adenocarcinoma of the esophagogastric junction (AEGJ) in Japan.

Methods We investigated the clinicopathological characteristics, treatment outcomes, and prognosis for 816 patients with esophagogastric junctional and gastric adenocarcinoma who received first-line chemotherapy between 2004 and 2009.

Results Of 816 patients, $82(10 \%)$ had AEGJ. The patients with AEGJ had significantly more lung and lymph
\end{abstract}

A. Kawano $\cdot$ T. E. Nakajima $\cdot$ S. Iwasa $\cdot$ K. Kato ·

T. Hamaguchi · Y. Yamada · Y. Shimada

Gastrointestinal Oncology Division, National Cancer Center

Hospital, 5-1-1 Tsukiji, Chuou-ku, Tokyo 104-0045, Japan

e-mail: akawano@east.ncc.go.jp

\section{A. Kawano $\cdot$ H. Fujii}

Clinical Oncology Division, Jichi Medical University Hospital, 3311-1 Yakushiji, Shimotsuke, Tochigi 329-0498, Japan

\section{A. Kawano}

6-5-1 Kashiwanoha, Kashiwa, Chiba 277-8577, Japan

T. E. Nakajima ( $\square)$

Department of Clinical Oncology, St. Marianna University,

School of Medicine, 2-16-1 Sugao, Miyamae-ku, Kawasaki,

Kanagawa 216-8511, Japan

e-mail: tnakajima@marianna-u.ac.jp

I. Oda

Endoscopy Division, National Cancer Center Hospital,

5-1-1 Tsukiji, Chuou-ku, Tokyo 104-0045, Japan

N. Hokamura

Esophageal Surgery Division, National Cancer Center Hospital,

5-1-1 Tsukiji, Chuou-ku, Tokyo 104-0045, Japan node metastasis, but less peritoneal metastasis, than those with gastric adenocarcinoma (GAC). The objective response rate to first-line chemotherapy was $23.3 \%$ for patients with AEGJ and $22.6 \%$ in patients with GAC $(p=0.90)$. The median survival was 13.0 months in AEGJ and 11.8 months in GAC $(p=0.445)$. In no patient was tumor site a significant prognostic factor $(p=0.472)$. In patients with AEGJ, ECOG PS $\geq 2$, presence of liver metastasis, and absence of lung metastasis were significantly associated with poor prognosis.

Conclusions No significant differences were observed in treatment outcomes between advanced AEGJ and GAC. Therefore, the same chemotherapy regimen can be given as a treatment arm in future Japanese clinical trials to both patients with inoperably advanced or recurrent AEGJ and those with GAC.

Keywords Siewert classification .

Esophagogastric junction - Adenocarcinoma .

Chemotherapy $\cdot$ Prognosis

\section{Introduction}

Adenocarcinoma of the esophagogastric junction (AEGJ) or lower esophagus is one of the most rapidly increasing malignant diseases in the West and appears to have a different etiology from distal gastric cancer [1-4]. In contrast, the incidence of AEGJ is unchanged or only gradually increasing in the East [5-7], and its clinicopathological features have not yet been elucidated, especially in advanced, nonresectable tumors.

From clinical trials for advanced cancers of the esophagus and the stomach, the current status of AEGJ is variable; it may be treated as either esophageal or gastric cancer, or be 
excluded from the trial altogether. Chau et al. [8] studied 1,775 patients with advanced esophagogastric cancer, including 457 with AEGJ, who had been treated with chemotherapy in four Western randomized trials. That study found no difference in overall survival (OS), response rates, or toxicities by tumor location.

In Japanese randomized trials for advanced esophageal or gastric cancer, AEGJ has not been specifically examined because of its rarity [9-13]. There is currently no standard chemotherapy regimen for AEGJ, and it is usually treated as a gastric cancer with fluoropyrimidine and platinum.

In this study, we retrospectively investigated clinicopathological features and treatment outcomes associated with advanced AEGJ in Japanese patients treated at a highvolume cancer center, examining whether AEGJ warrants a separate clinical approach in future clinical trials.

\section{Patients and methods}

\section{Patients}

We retrospectively analyzed patients with inoperably advanced or recurrent gastric and esophageal cancer who had received palliative therapy between January 2004 and December 2009 at the National Cancer Center Hospital in Tokyo. The eligibility criteria for this study were as follows: (1) histologically confirmed adenocarcinoma; (2) treatment with first-line chemotherapy in our hospital; and (3) availability of clinicopathological data at the beginning of the first-line chemotherapy. Carcinomas in remnant stomach after partial gastrectomy were excluded. Of 1,395 patients who received palliative therapy in our hospital between 2004 and 2009, 816 patients were enrolled in this study (Fig. 1). All endpoints were updated in March 2011. Median follow-up time was 11.1 months (range, 0.8-82.0 months), and median follow-up time for the surviving patients was 19.0 months.

\section{Clinicopathological data}

Performance status (PS) at the beginning of first-line chemotherapy was evaluated according to the Eastern Cooperative Oncology Group criteria. Clinical tumor response was assessed according to Response Evaluation Criteria in Solid Tumors (RECIST ver. 1.0). The histological type of the primary tumor was evaluated by using a biopsy specimen of inoperably advanced cases and the surgical specimen of recurrent cases. Histological type was determined according to the Japanese classification for gastric carcinoma for the predominant histological type [14]. Papillary, well- or moderately differentiated adenocarcinoma was defined as the intestinal type, whereas poorly differentiated

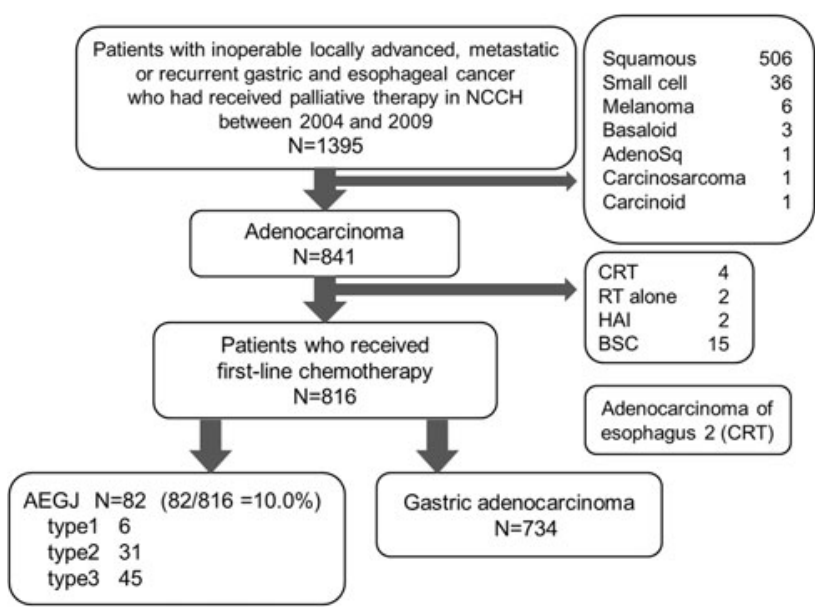

Fig. 1 Trial profile. $\mathrm{NCCH}$ National Cancer Center Hospital, Ade$n o S q$ adenosquamous carcinoma, $C R T$ chemoradiotherapy, $R T$ radiotherapy, $H A I$ hepatic arterial infusion therapy, $B S C$ best supportive care, AEGJ adenocarcinoma of esophagogastric junction

adenocarcinoma or signet-ring cell carcinoma was defined as the diffuse type. Mucinous adenocarcinoma was classified as intestinal or diffuse depending on the secondary predominant histological type.

Baseline characteristics at the beginning of first-line chemotherapy such as age, sex, PS, and laboratory data were evaluated. The following clinicopathological factors were also evaluated: disease status (inoperably advanced or recurrent), histopathology (intestinal or diffuse), metastatic site at the beginning of first-line chemotherapy (liver, peritoneum, lung, bone, abdominal lymph node, mediastinal lymph node, and cervical lymph node), number of metastatic sites, and response to first-line chemotherapy.

\section{AEGJ classification}

The tumor location of AEGJ was defined in accordance with Siewert's classification [15]. The Siewert subtypes were retrospectively determined by the following method. In recurrent patients, pathologists recorded the relationship between the tumor center and EGJ according to Siewert's classification, when diagnosing the surgically resected specimen. In inoperably advanced patients, two endoscopists retrospectively determined the relationship between the EGJ and the tumor center independently of each other.

Statistical analysis

Statistical analysis was performed using SPSS 17.0 statistical software (SPSS, Chicago, IL, USA). Comparison of categorical variables was tested by the Chi square test. OS was calculated from the date of the first diagnosis of inoperably advanced or recurrent gastric cancer to death from any cause that was scored as an event. Patients who were 
Table 1 Clinical findings of all patients
$A E G J$ adenocarcinoma of the esophagogastric junction, $G A C$ gastric adenocarcinoma, $L N$ lymph node, $F$ fluoropyrimidine (5-fluorouracil, S-1, capecitabine), $P$ platinum (cisplatin, oxaliplatin), NA not available

${ }^{\text {a }}$ Best overall response rate $=$ (complete response + partial response)

${ }^{\mathrm{b}}$ Disease control rate $=$ (complete response + partial response + stable disease)

\begin{tabular}{|c|c|c|c|}
\hline Factor & $\begin{array}{l}\text { AEGJ } \\
N=82\end{array}$ & $\begin{array}{l}\text { GAC } \\
N=734\end{array}$ & $p$ value \\
\hline Median age, years (range) & $62(24-85)$ & $63(19-84)$ & 0.085 \\
\hline Gender & & & $<0.001$ \\
\hline Male & $70(85 \%)$ & $458(62 \%)$ & \\
\hline Female & $12(15 \%)$ & $276(38 \%)$ & \\
\hline Performance status & & & 0.217 \\
\hline 0 & $38(46 \%)$ & $273(37 \%)$ & \\
\hline 1 & $40(49 \%)$ & $411(56 \%)$ & \\
\hline$\geq 2$ & $4(5 \%)$ & $48(6 \%)$ & \\
\hline Unknown & 0 & 2 & \\
\hline Disease status & & & 0.030 \\
\hline Inoperable & $47(57 \%)$ & $506(69 \%)$ & \\
\hline Recurrent & $35(43 \%)$ & $228(31 \%)$ & \\
\hline Tumor differentiation on histopathology & & & $<0.001$ \\
\hline Intestinal & $39(48 \%)$ & $195(27 \%)$ & \\
\hline Diffuse & $44(52 \%)$ & $437(77 \%)$ & \\
\hline Not classified & 0 & 21 & \\
\hline Number of metastatic sites & & & $<0.001$ \\
\hline 1 & $45(55 \%)$ & $563(77 \%)$ & \\
\hline$\geq 2$ & $37(45 \%)$ & $171(23 \%)$ & \\
\hline \multicolumn{4}{|l|}{ Metastatic/recurrent sites } \\
\hline Liver & $23(28 \%)$ & $196(27 \%)$ & 0.794 \\
\hline Peritoneum & $21(27 \%)$ & $402(55 \%)$ & $<0.001$ \\
\hline Lung & $21(27 \%)$ & $40(5 \%)$ & $<0.001$ \\
\hline Bone & $4(5 \%)$ & $44(6 \%)$ & 0.684 \\
\hline Abdominal LN & $34(41 \%)$ & $197(27 \%)$ & 0.010 \\
\hline Mediastinal LN & $17(21 \%)$ & $22(3 \%)$ & $<0.001$ \\
\hline Cervical LN & $7(9 \%)$ & $30(4 \%)$ & 0.066 \\
\hline First-line chemotherapy regimen & & & NA \\
\hline F alone & $31(38 \%)$ & $333(45 \%)$ & \\
\hline $\mathrm{F}+\mathrm{P}$ & $24(29 \%)$ & $128(17 \%)$ & \\
\hline $\mathrm{F}+\mathrm{P} \pm$ anti-angiogenetic agent & $2(2 \%)$ & $32(4 \%)$ & \\
\hline $\mathrm{F}+$ taxane & $11(13 \%)$ & $34(5 \%)$ & \\
\hline Irinotecan regimen & $9(11 \%)$ & $94(13 \%)$ & \\
\hline Taxane alone & $5(6 \%)$ & $25(3 \%)$ & \\
\hline Best overall response & & & NA \\
\hline Complete response & 0 & $9(1 \%)$ & \\
\hline Partial response & $17(21 \%)$ & $142(20 \%)$ & \\
\hline Stable disease & $32(39 \%)$ & $323(44 \%)$ & \\
\hline Progressive disease & $24(29 \%)$ & $193(26 \%)$ & \\
\hline Not evaluable & $9(11 \%)$ & $67(9 \%)$ & \\
\hline Best overall response rate ${ }^{a}$ & $23.3 \%$ & $22.6 \%$ & 0.90 \\
\hline Disease control rate ${ }^{\mathrm{b}}$ & $67.1 \%$ & $71.1 \%$ & 0.48 \\
\hline
\end{tabular}

alive were censused at the last follow-up date. Survival curves were derived from Kaplan-Meier estimates, and the curves were compared by log-rank tests. A prognostic model was established by searching all variables that significantly influenced OS at a level of $p$ values $<0.05$ in the univariate analysis. Multivariate analysis for OS was performed using stepwise Cox's proportional hazard regression model (entry probability 0.05 , removal probability 0.1 ). All the tests were two sided, and $p$ values $<0.05$ were considered significant. 


\section{Results}

Figure 1 shows the screening process of this study; 816 patients were finally enrolled and analyzed. Eighty-two (10\%) patients had AEGJ and 734 patients had gastric adenocarcinoma (GAC). Among the 82 patients with AEGJ, $6(7 \%)$ were classified as Siewert type I, 31 (38\%) as type II, and $45(55 \%)$ as type III. Table 1 shows the baseline clinicopathological characteristics. There were significantly more males $(p<0.001)$, recurrent status $(p=0.03)$, intestinal differentiated tumors on histopathology ( $p<0.001)$, lung metastasis $(p<0.001)$, and lymph node metastasis $(p<0.001)$ in patients with AEGJ than in those with GAC. On the other hand, there was significantly more peritoneal metastasis in patients with GAC than in those with AEGJ $(p<0.001)$. There was no difference among the two groups in the first-line chemotherapy regimen. The objective response (complete and partial response) rate was $23.3 \%$ for patients with AEGJ and $22.6 \%$ in patients with GAC ( $p=0.90)$. The disease control (complete and partial response plus stable disease) rate was $67.1 \%$ for patients with AEGJ and 71.1 \% in patients with GAC ( $p=0.48)$. In the patients treated by the $\mathrm{F}+\mathrm{P}$ regimen, the objective response rate was $27.3 \%$ in patients with AEGJ and $29.2 \%$ in patients with GAC, and the disease control rate was 72.7 and $88.3 \%$, respectively. There were also no significant differences between two groups.

At the time of data cutoff, $668(82 \%)$ patients had died. Median survival was 13.0 months [95\% confidence interval (CI), 9.0-16.9 months] in AEGJ and 11.8 months (95\% CI, 10.9-12.7 months) in GAC. Figure 2 shows that there were no significant differences in OS between AEGJ and GAC (log-rank, $p=0.445)$. In the patients treated by the $\mathrm{F}+\mathrm{P}$ regimen, which is the standard therapy for gastric and esophageal cancer, the survival time was not

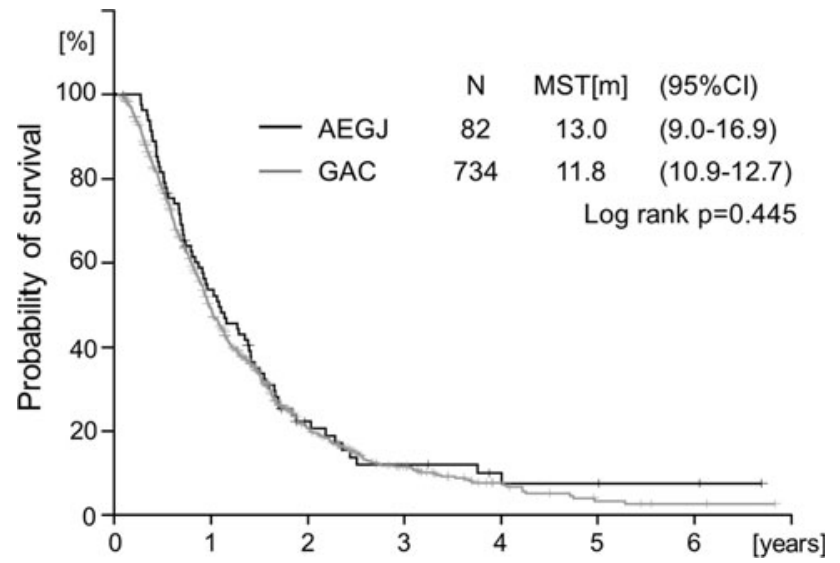

Fig. 2 Overall survival curves according to tumor site. AEGJ adenocarcinoma of esophagogastric junction, $M S T$ median survival time, $C I$ confidence interval significantly different between the patients with AEGJ and those with GAC (log-rank, $p=0.352$ ).

In no patient was tumor site (EGJ or gastric) a significant prognostic factor $(p=0.472)$. The results of univariate analysis of clinicopathological variables for prognostic factors in patients with AEGJ and GAC are shown in Table 2. In univariate analysis, four variables were significantly associated with poor survival time in patients with GAC: ECOG PS $\leq 2$, inoperably advanced disease status, diffuse histopathology, and two or more metastatic sites. On the other hands, three variables were significantly associated with poor survival time in those with AEGJ: ECOG PS $\geq 2$, the presence of liver metastasis, and absence of lung metastasis. The independent prognostic factors identified by the multivariate analysis are all significant prognostic factors identified by univariate analysis. The results of multivariate analysis for prognostic factors in patients with AEGJ and GAC are shown in Table 3. Poor PS was an independent prognostic factor in patients with both AEGJ and GAC. However, there were some differences in prognostic factors between AEGJ and GAC. In patients with GAC, inoperably advanced disease status, diffuse histopathology, and increasing number of metastatic sites influenced survival. The presence of liver metastasis and absence of lung metastasis were also associated with poor prognosis in those with AEGJ.

\section{Discussion}

This study first reported from Asia that the clinicopathological characteristics of inoperably advanced or recurrent AEGJ, including sex, tumor location, and histological type, were similar to those of operable AEGJ previously reported in Japan and also in Western countries [8, 16-20].

Lung metastasis was diagnosed in $7-41 \%$ of the patients with advanced esophageal cancer, and its frequency was high compared with about $5 \%$ of patients diagnosed with advanced gastric cancer [12, 21-23]. Because of invasion to the esophagus, AEGJ may have the same drainage system of a vein as lower esophageal cancer. Additionally, it was reported that the mediastinal lymph node metastasis rate of gastric cancer depended on the length of esophageal invasion, and a length of more than 2-3 cm was a risk factor [22, 24]. On the other hand, patients with AEGJ had little peritoneal metastasis because of its anatomical location and histology. Most type I and II AEGJs are not fully covered by the peritoneum. Moreover, peritoneal metastasis was more frequent in patients with diffuse-type histopathology compared to those with intestinal type, the primary histopathological type of AEGJ.

Our data showing that patients with AEGJ have significantly more lung metastasis, more mediastinal lymph node 
Table 2 Univariate analyses on survival
$A E G J$ adenocarcinoma of esophagogastric junction, $G A C$ gastric adenocarcinoma, $N$ number of patients, $H R$ hazard ratio, $C I$ confidence interval

\begin{tabular}{|c|c|c|c|c|c|c|c|c|}
\hline Factor & $N$ & HR & $95 \% \mathrm{CI}$ & $p$ value & $N$ & $\mathrm{HR}$ & $95 \%$ CI & $p$ value \\
\hline \multicolumn{9}{|l|}{ Age } \\
\hline$<60$ & 36 & 1 & & & 270 & 1 & & \\
\hline$\geq 60$ & 46 & 1.13 & $0.70-1.82$ & 0.630 & 464 & 1.16 & $0.99-1.38$ & 0.074 \\
\hline \multicolumn{9}{|l|}{ Sex } \\
\hline Male & 70 & 1 & & & 458 & 1 & & \\
\hline Female & 12 & 1.62 & $0.82-3.19$ & 0.166 & 276 & 1.09 & $0.93-1.29$ & 0.293 \\
\hline \multicolumn{9}{|c|}{ Performance status } \\
\hline $0-1$ & 78 & 1 & & & 681 & 1 & & \\
\hline$\geq 2$ & 4 & 16.3 & $4.09-65.2$ & $<0.001$ & 53 & 3.30 & $2.44-4.47$ & $<0.001$ \\
\hline \multicolumn{9}{|c|}{ Disease status } \\
\hline Inoperable & 47 & 1 & & & 506 & 1 & & \\
\hline Recurrent & 35 & 0.77 & $0.47-1.26$ & 0.300 & 228 & 0.73 & $0.61-0.87$ & $<0.001$ \\
\hline \multicolumn{9}{|l|}{ Pathology } \\
\hline Intestinal & 39 & 1 & & & 195 & 1 & & \\
\hline Diffuse & 43 & 1.57 & $0.97-2.54$ & 0.067 & 521 & 1.29 & $1.07-1.56$ & 0.008 \\
\hline \multicolumn{9}{|c|}{ Number of metastases } \\
\hline 1 & 42 & 1 & & & 546 & 1 & & \\
\hline$\geq 2$ & 40 & 1.42 & $0.88-2.28$ & 0.153 & 188 & 1.47 & $1.23-1.77$ & $<0.001$ \\
\hline \multicolumn{9}{|c|}{ Liver metastasis } \\
\hline Absent & 59 & 1 & & & 538 & 1 & & \\
\hline Present & 23 & 1.97 & $1.19-3.27$ & 0.009 & 196 & 1.16 & $0.97-1.39$ & 0.104 \\
\hline \multicolumn{9}{|c|}{ Peritoneal metastasis } \\
\hline Absent & 61 & 1 & & & 332 & 1 & & \\
\hline Present & 21 & 1.22 & $0.71-2.08$ & 0.467 & 402 & 1.00 & $0.85-1.18$ & 0.991 \\
\hline \multicolumn{9}{|c|}{ Lung metastasis } \\
\hline Absent & 61 & 1 & & & 694 & 1 & & \\
\hline Present & 21 & 0.37 & $0.20-0.68$ & 0.001 & 40 & 0.90 & $0.63-1.29$ & 0.563 \\
\hline \multicolumn{9}{|c|}{ Bone metastasis } \\
\hline Absent & 78 & 1 & & & 690 & 1 & & \\
\hline Present & 4 & 2.07 & $0.49-8.78$ & 0.322 & 44 & 1.35 & $0.97-1.89$ & 0.076 \\
\hline \multicolumn{9}{|c|}{ Lymph node metastasis } \\
\hline Absent & 36 & 1 & & & 518 & 1 & & \\
\hline Present & 46 & 1.16 & $0.71-1.89$ & 0.543 & 216 & 1.15 & $0.97-1.37$ & 0.118 \\
\hline \multicolumn{9}{|c|}{ Abdominal lymph node } \\
\hline Absent & 48 & 1 & & & 537 & 1 & & \\
\hline Present & 34 & 0.92 & $0.57-1.50$ & 0.737 & 197 & 1.14 & $0.95-1.37$ & 0.145 \\
\hline \multicolumn{9}{|c|}{ Mediastinal lymph node } \\
\hline Absent & 65 & 1 & & & 712 & 1 & & \\
\hline Present & 17 & 1.29 & $0.71-2.34$ & 0.400 & 22 & 1.28 & $0.81-2.03$ & 0.286 \\
\hline \multicolumn{9}{|c|}{ Cervical lymph node } \\
\hline Absent & 75 & 1 & & & 704 & 1 & & \\
\hline Present & 7 & 2.32 & $0.98-5.47$ & 0.055 & 30 & 1.16 & $0.77-1.73$ & 0.480 \\
\hline
\end{tabular}

metastasis, and less peritoneal metastasis than patients with gastric cancer are consistent with those of previous reports.

The median survival time of patients with advanced AEGJ was 13.0 months, and there was no significant difference in survival between the patients with AEGJ and those with $\operatorname{GAC}(p=0.445)$ in our analysis. In the patients treated with the $\mathrm{F}+\mathrm{P}$ chemotherapy regimen, the OS was not significantly different between AEGJ and GAC ( $p=0.352$ ). These survival data for the patients receiving $\mathrm{F}+\mathrm{P}$ is almost the same as those for inoperable gastric cancer patients who were enrolled and received $\mathrm{F}+\mathrm{P}$ in Japanese phase III trials [11-13]. 
Table 3 Multivariate analysis on survival

\begin{tabular}{|c|c|c|c|c|c|c|}
\hline \multirow[t]{2}{*}{ Factor } & \multicolumn{3}{|c|}{ Patients with AEGJ } & \multicolumn{3}{|c|}{ Patients with GAC } \\
\hline & HR & $95 \% \mathrm{CI}$ & $p$ value & HR & $95 \% \mathrm{CI}$ & $p$ value \\
\hline \multicolumn{7}{|c|}{ Performance status } \\
\hline $0-1$ & 1 & & & 1 & & \\
\hline$\geq 2$ & 10.56 & $2.68-41.86$ & 0.001 & 3.15 & $2.32-4.27$ & $<0.001$ \\
\hline \multicolumn{7}{|c|}{ Disease status } \\
\hline Inoperable & & & & 1 & & \\
\hline Recurrent & & & $\mathrm{NE}$ & 0.76 & $0.63-0.91$ & 0.002 \\
\hline \multicolumn{7}{|l|}{ Pathology } \\
\hline Intestinal & & & & 1 & & \\
\hline Diffuse & & & $\mathrm{NE}$ & 1.32 & $1.09-1.59$ & 0.004 \\
\hline \multicolumn{7}{|c|}{ Number of metastases } \\
\hline 1 & & & & 1 & & \\
\hline$\geq 2$ & & & $\mathrm{NE}$ & 1.45 & $1.21-1.75$ & $<0.001$ \\
\hline \multicolumn{7}{|c|}{ Liver metastasis } \\
\hline Absent & 1 & & & & & \\
\hline Present & 2.22 & $1.31-3.78$ & 0.003 & & & $\mathrm{NE}$ \\
\hline \multicolumn{7}{|c|}{ Lung metastasis } \\
\hline Absent & 1 & & & & & \\
\hline Present & 0.33 & $0.18-0.63$ & 0.001 & & & $\mathrm{NE}$ \\
\hline
\end{tabular}

We identified poor PS, the presence of liver metastasis, and absence of lung metastasis as baseline prognostic factors in patients with inoperably advanced or recurrent AEGJ. Several studies have identified prognostic factors for patients with metastatic gastric cancer who received first-line chemotherapy: poor PS, the presence of liver, peritoneal, or bone metastases, microscopically scirrhous type tumors, and number of metastatic sites [25, 26]. Chau et al. [27] also elucidated that poor PS and the presence of liver or peritoneal metastases was associated with poor prognosis for patients with advanced esophageal, EGJ, and gastric cancer. The prognostic factors in AEGJ identified in our report are compatible with the prognostic factors reported in EGJ and gastric cancer.

Chau et al. [8] reported that the survival curves of patients with advanced AEGJ and GAC almost overlapped and so it might not be necessary to distinguish patients with advanced esophagogastric adenocarcinoma according to primary tumor origin. Our results were consistent with this report. We consider that the same chemotherapy can be given to both patients with inoperably advanced or recurrent AEGJ and those with GAC in the clinical practice in Japan, and Japanese future trials on gastric cancer chemotherapy can include both subgroups.

This study had several limitations because it was a retrospective, single-institution study. First, because the selection of chemotherapy regimen in patients with AEGJ was not standardized, the study included several chemotherapy regimens and tumor location itself might have influenced regimen selection, although differences were not statistically significant. Second, disease progression was judged by the investigators in this study.

In conclusion, we identified that the incidence, characteristics, treatment outcomes, and prognosis for patients with AEGJ showed no significant differences compared with those for patients with GAC. We consider that Japanese future trials on gastric cancer chemotherapy can include both subgroups.

\section{References}

1. Botterweck AA, Schouten LJ, Volovics A, Dorant E, van Den Brandt PA. Trends in incidence of adenocarcinoma of the oesophagus and gastric cardia in ten European countries. Int $\mathbf{J}$ Epidemiol 2000;29:645-54.

2. Devesa SS, Blot WJ, Fraumeni JF Jr. Changing patterns in the incidence of esophageal and gastric carcinoma in the United States. Cancer (Phila). 1998;83:2049-53.

3. Parfitt JR, Miladinovic Z, Driman DK. Increasing incidence of adenocarcinoma of the gastroesophageal junction and distal stomach in Canada: an epidemiological study from 1964-2002. Can J Gastroenterol. 2006;20:271-6.

4. Stein HJ, Feith M, Siewert JR. Cancer of the esophagogastric junction. Surg Oncol. 2000;9:35-41.

5. Chung JW, Lee GH, Choi KS, Kim DH, Jung KW, Song HJ, et al. Unchanging trend of esophagogastric junction adenocarcinoma in Korea: experience at a single institution based on Siewert's classification. Dis Esophagus. 2009;22:676-81.

6. Deans C, Yeo MS, Soe MY, Shabbir A, Ti TK, So JB. Cancer of the gastric cardia is rising in incidence in an Asian population and 
is associated with adverse outcome. World J Surg. 2011; 35:617-24

7. Kusano C, Gotoda T, Khor CJ, Katai H, Kato H, Taniguchi H, et al. Changing trends in the proportion of adenocarcinoma of the esophagogastric junction in a large tertiary referral center in Japan. J Gastroenterol Hepatol. 2008;23:1662-5.

8. Chau I, Norman AR, Cunningham D, Oates J, Hawkins R, Iveson $\mathrm{T}$, et al. The impact of primary tumour origins in patients with advanced oesophageal, oesophago-gastric junction and gastric adenocarcinoma-individual patient data from 1775 patients in four randomised controlled trials. Ann Oncol. 2009;20:885-91.

9. Iizuka T, Kakegawa T, Ide H, Ando N, Watanabe H, Tanaka O, et al. Phase II evaluation of cisplatin and 5-fluorouracil in advanced squamous cell carcinoma of the esophagus: a Japanese Esophageal Oncology Group Trial. Jpn J Clin Oncol. 1992;22: $172-6$.

10. Hayashi K, Ando N, Watanabe H, Ide H, Nagai K, Aoyama N, et al. Phase II evaluation of protracted infusion of cisplatin and 5-fluorouracil in advanced squamous cell carcinoma of the esophagus: a Japan Esophageal Oncology Group (JEOG) Trial (JCOG9407). Jpn J Clin Oncol. 2001;31:419-23.

11. Boku N, Yamamoto S, Fukuda H, Shirao K, Doi T, Sawaki A, et al. Fluorouracil versus combination of irinotecan plus cisplatin versus S-1 in metastatic gastric cancer: a randomised phase 3 study. Lancet Oncol 2009;10:1063-69.

12. Koizumi W, Narahara H, Hara T, Takagane A, Akiya T, Takagi $\mathrm{M}$, et al. S-1 plus cisplatin versus S-1 alone for first-line treatment of advanced gastric cancer (SPIRITS trial): a phase III trial. Lancet Oncol. 2008;9:215-21.

13. Ohtsu A, Shimada Y, Shirao K, Boku N, Hyodo I, Saito H, et al. Randomized phase III trial of fluorouracil alone versus fluorouracil plus cisplatin versus uracil and tegafur plus mitomycin in patients with unresectable, advanced gastric cancer: the Japan Clinical Oncology Group Study (JCOG9205). J Clin Oncol. 2003; 21:54-9.

14. Japanese Gastric Cancer Association. Japanese classification of gastric carcinoma, 2nd English edition. Gastric Cancer 1998;1: 10-24.

15. Siewert JR, Stein HJ. Carcinoma of the cardia: carcinoma of the gastroesophageal junction-classification, pathology and extent of resection. Dis Esophagus. 1996;9:173-82.

16. Van Cutsem E, Moiseyenko VM, Tjulandin S, Majlis A, Constenla M, Boni C, et al. Phase III study of docetaxel and cisplatin plus fluorouracil compared with cisplatin and fluorouracil as firstline therapy for advanced gastric cancer: a report of the V325 Study Group. J Clin Oncol. 2006;24:4991-7.
17. Cunningham D, Starling N, Rao S, Iveson T, Nicolson M, Coxon $\mathrm{F}$, et al. Capecitabine and oxaliplatin for advanced esophagogastric cancer. N Engl J Med. 2008;358:36-46.

18. Ajani JA, Rodriguez W, Bodoky G, Moiseyenko V, Lichinitser M, Gorbunova V, et al. Multicenter phase III comparison of cisplatin/S-1 with cisplatin/infusional fluorouracil in advanced gastric or gastroesophageal adenocarcinoma study: the FLAGS trial. J Clin Oncol. 2010;28:1547-53.

19. Kodera Y, Yamamura Y, Shimizu Y, Torii A, Hirai T, Yasui K, et al. Adenocarcinoma of the gastroesophageal junction in Japan: relevance of Siewert's classification applied to 177 cases resected at a single institution. J Am Coll Surg. 1999;189:594-601.

20. Hasegawa S, Yoshikawa T, Cho H, Tsuburaya A, Kobayashi O. Is adenocarcinoma of the esophagogastric junction different between Japan and western countries? The incidence and clinicopathological features at a Japanese high-volume cancer center. World J Surg. 2009;33:95-103.

21. Quint LE, Hepburn LM, Francis IR, Whyte RI, Orringer MB. Incidence and distribution of distant metastases from newly diagnosed esophageal carcinoma. Cancer (Phila). 1995;76: $1120-5$.

22. Kodama I, Kofuji K, Yano S, Shinozaki K, Murakami N, Hori H, et al. Lymph node metastasis and lymphadenectomy for carcinoma in the gastric cardia: clinical experience. Int Surg. 1998; 83:205-9.

23. Hasegawa S, Yoshikawa T. Adenocarcinoma of the esophagogastric junction: incidence, characteristics, and treatment strategies. Gastric Cancer. 2010;13:63-73.

24. Nunobe S, Ohyama S, Sonoo H, Hiki N, Fukunaga T, Seto Y, et al. Benefit of mediastinal and para-aortic lymph-node dissection for advanced gastric cancer with esophageal invasion. J Surg Oncol. 2008;97:392-5.

25. Lee J, Lim T, Uhm JE, Park KW, Park SH, Lee SC, et al. Prognostic model to predict survival following first-line chemotherapy in patients with metastatic gastric adenocarcinoma. Ann Oncol. 2007;18:886-91.

26. Yoshida M, Ohtsu A, Boku N, Miyata Y, Shirao K, Shimada Y, et al. Long-term survival and prognostic factors in patients with metastatic gastric cancers treated with chemotherapy in the Japan Clinical Oncology Group (JCOG) study. Jpn J Clin Oncol. 2004; 34:654-9.

27. Chau I, Norman AR, Cunningham D, Waters JS, Oates J, Ross PJ. Multivariate prognostic factor analysis in locally advanced and metastatic esophago-gastric cancer: pooled analysis from three multicenter, randomized, controlled trials using individual patient data. J Clin Oncol. 2004;22:2395-403. 